\title{
A single-station spectral model of the monthly median foF2 over Chongqing, China
}

\author{
Tong Xu $\left({ }^{1}\right)$, Zhensen Wu $\left({ }^{1}\right)$, Jian Wu $\left({ }^{2}\right)$, Guohui Wei $\left(^{3}\right)$ and Jian Feng $\left({ }^{4}\right)$ \\ (') School of Science, Xidian University, Xi'an, China \\ $\left.{ }^{2}{ }^{2}\right)$ National Key Laboratory of Electromagnetic Environment, China Research Institute of \\ Radiowave Propagation, Beijing, China \\ $\left(^{3}\right)$ School of Science, Southwest Petroleum University, Chengdu, China \\ $\left(^{4}\right)$ China Research Institute of Radiowave Propagation, Qingdao, China
}

\begin{abstract}
Hourly values of the critical frequency of the ionospheric F region, foF2, obtained at Chongqing ionospheric observatory (geographic $29.5^{\circ} \mathrm{N}, 106.4^{\circ} \mathrm{E}$ ), China, during the interval of 1977 to 1997 (solar cycle 21 and 22) have been used to investigate the dependence of the monthly median foF 2 on solar activity and geomagnetic activity, and to construct single-station model (SSM) using Fourier expansion. The results of the present analysis show that there is a significant nonlinear relationship between monthly median foF2 and sunspot number R especially at daytime in each month and nighttime in summer. Furthermore, introducing geomagnetic index Ap further improves the description of variation of monthly median foF2, and the standard deviations decrease also dependently on time and month. Thus the complex influence of solar activity and geomagnetic activity may be approximately expressed by a general multiple nonlinear function at Chongqing station, China. The basic aim of this paper is to show that considering the nonlinear influence both of solar activity and geomagnetic activity improves the monthly median model. The SSM is in good agreement with observations, with standard deviation $0.65 \mathrm{MHz}$, while IRI model has a lower accuracy with standard deviation $0.96 \mathrm{MHz}$.
\end{abstract}

Key words solar cycle variation - single station model - geomagnetic index - monthly median foF 2

\section{Introduction}

Among ionospheric characteristic parameters, the critical frequency of the $\mathrm{F}$ region, foF2 is one of the most important ones. Over decades, great efforts have been made to study

Mailing address: Dr. Xu Tong, School of Science, Xidian University, Box 48, Xi' an, Shaanxi, 710071, China; email: xutong1104@126.com the statistical model of foF2. An excellent review of many available models has been presented recently by Bilitza (2002) and Zolesi and Cander (2000). Among these models, the International Reference ionosphere (IRI) model, a global statistic model, is most widely used, and is recognized as the standard specification of ionospheric parameters by the Committee on Space Research (COSPAR) and the International Union of Radio Science (URSI).

However, many research workers have developed the single-station model (SSM) for a particular station (Moraitis et al., 1991; Sizun, 1991; Dick and Bradley, 1992; Kouris et al., 1993; Pancheva and Mukhtarov, 1998; Liu et al., 2004). The basic advantages of the SSM are: i) more accurate results for a particular ionosonde station than the global one, 
and ii) the current updating of a SSM is usually a very easy process (Pancheva and Mukhtarov, 1996). It is well known that the foF2 depends strongly on solar activity. At middle and low latitudes, the primary source of ionization in the $\mathrm{F}$ region is the solar extreme ultraviolet (EUV) irradiances. Thus ionosphere empirical models depend on how to describe the relationship between foF 2 and thesolar activity index. However, measurements of EUV have been rather meager, especially in the past. Traditionally sunspot number $\mathrm{R}$ is considered as an index of solar activity, although other indices, solar or ionospherically derived (Liu et al., 1983; Caruana, 1990; Mikhailov and Mikhailov, 1995), are also used. The sunspot number is convenient for use because of its long series of observations and its characteristics of reliability and predictability.

The monthly median values of the ionospheric characteristic are frequently presented with linear dependence on $\mathrm{R}$ for every hour in the day and every month in the year, e.g. the international reference ionosphere (Bilitza, 2001), ionospheric regional model (Zolesi et al., 1993; De Franceschi and Desantis, 1994) as well as single-station model (Pancheva and Mukhtarov, 1996; Holt et al., 2002). For low and medium sunspot numbers, the relationship is reasonably linear, nevertheless at large sunspot numbers, foF 2 seems to show saturation effect (Huang, 1963; Kane, 1992). To take into account this behavior, a second-degree relationship between foF 2 and solar activity indices is used frequently (Sizun, 1992; Xenos et al., 1996; Pancheva and Mukhtarov, 1998). This «hyseresis» effect depends on latitude (Sethi et al., 2002) and historical solar activity (Rao and Rao, 1969; Liu et al., 2004). In fact Liu et al. (2004) and Sethi et al. (2002) claimed that for low-latitudes, a second-degree relationship made much improvement, while, for European stations, Kouris and Nissopoulos (1994) have demonstrated that a second-degree equation is needed especially during evening and night hours in the winter and summer-time.

Moreover the variations of foF 2 are complex and cannot be described considering only sunspot number. The ionosphere variations are due to the influence of the solar and geomagnetic activities as well as other sources (Forbes et al., 2000; Zhang et al., 2004). For the same $\mathrm{R}$, foF2 may show different values during the ascending and descending of the cycle (Rao and Rao, 1969) and is attributed to possible geomagnetic storm effect (Kane, 1992), a view also shared by Apostolov et al. (1994). Pancheva and Mukhtarov (1996) developed a single-station spectral model over Sofia with $R$ and $K_{R}$ and demonstrated that in many cases the increase in the monthly standard deviation coincided with the increase in the geomagnetic activity index aa. Kane (1992) argued that longterm prediction models need to take into account not just R but some solar index and geomagnetic index as two key parameters to which not much attention has been paid to date. One objective of this work is to assess both the solar activity and geomagnetic activity dependence of the monthly median foF 2 over Chongqing.

Because of the advantages of SSM and lack of SSM for foF2 yet at Chongqing or in the Chinese subcontinent, we develop a spectral model both taking sunspot number $\mathrm{R}$ and geomagnetic index Ap into account based on the data from 1977 to 1997 over Chongqing. The data description is presented in Section 2; the relationship between foF2 and solar activity and geomagnetic activity are considered in Section 3; the model and results are presented in Section 4. The last section contains some discussions and conclusions.

\section{Data set}

The time series of monthly median hourly values of foF2 for the time interval 1977-1997 (solar cycle 21 and 22), or 21years, was processed at Chongqing station (geographic $29.5^{\circ} \mathrm{N}, 106.4^{0} \mathrm{E}$ ), which, located in central China, is away from the northern crest of equatorial anomaly in East Asia. It has significant values for studying ionospheric dynamics in the equatorial anomaly region. Because this observatory is in the transition area of middle latitudes to low latitudes, the foF2 variation is expected to be dependent both on nonlinear solar activity and geomagnetic activity. 


\section{Solar activity and geomagnetic activity dependence of foF 2}

We first consider the statistical relationship between foF 2 and sunspot number R and geomagnetic Ap before constructing spectral model. Ionospheric electron density is mainly due to the ionization of neutral atmosphere by solar radiation, thus electron density of $\mathrm{F}$ region increases with the increasing of solar activities (Balan et al., 1994; Kouris et al., 1998; Richards, 2001; Sethi et al., 2002), whereas the monthly median foF2 increase with solar activities in a rather complicated way (Kouris et al., 1998; Richards, 2001). Monthly median foF2 linearly increases with the long-term solar activity, but saturates at extremely high solar epochs (Kane, 1992; Liu et al., 2003). Many researchers have shown that the nonlinear relationship between foF2 and solar activity.

We use regression methods to study the solar activity dependence of foF 2 over Chongqing. Fist regression model is a linear approximation to describe the relationship between sunspot number $\mathrm{R}$ and foF2

$$
f_{0} F 2_{h, m}=a_{0 h, m}+a_{1 h, m} \cdot R
$$

$\mathrm{h}$ and $\mathrm{m}$ are the hour and the month of interest while $\mathrm{a}_{0}$ and $\mathrm{a}_{1}$ are two matrices of $24 \times 12=288$ coefficients for each hour of day and each month of the year, and R is twelve-month running mean values of sunspot number. The linear model has been applied largely (Dominici and Zolesi, 1987; Holt et al. 2002).The regression employing the method of least squares was performed between the measured foF 2 data of each hour of each month, and the results at local time noon $(12 \mathrm{~h})$ and midnight $(0 \mathrm{~h})$ are presented in fig. 1 and fig. 2 with dotted lines.

The second-degree regression is expected to make improvement, and the quadratic relationship between $R$ and foF 2 is described as

$$
f_{0} F 2_{h, m}=b_{0 h, m}+b_{1 h, m} \cdot R+b_{2 h, m} \cdot R^{2}
$$

where $b_{0}, b_{1}$ and $b_{2}$ also are the coefficients at specified time $h$ and month $m$. Sample fit results are also represented with solid lines in fig. 1 and fig. 2.
As shown in fig. 1, we find that the foF 2 is almost entirely dependent on nonlinear solar activity, while in fig. 2 an evident non linear dependence is observed only in summer (May, June and July) which is nearly consistent with the conclusions of Kouris and Nissopoulos (1994). Furthermore at noon in each month, $\mathrm{b}_{2}<0$, i.e. when sunspot number $\mathrm{R}$ reaches high enough, the monthly median foF2 decreases with the increasing solar activity. But at midnight in winter, $b_{2}>0$, indicating that foF2 increases with the increasing solar activity, i.e. the saturation effect is unobvious. Liu et al. (2004) firstly reported this phenomena based on the data from 1957 to 1991 at Wuhan station (geographic $30.6^{\circ} \mathrm{N}, 114.4^{\circ} \mathrm{E}$ ), China, which indicates that the phenomena may exist in low/middle-latitude in China but needs more ionosonde observations data to validate.

It is well known that ionosphere is principally determined by the level of both solar activity and geomagnetic perturbations, but similar studies for the geomagnetic index dependence of foF2 have been more limited, but some exist (Solé, 1988). Further significant improvement is expected from a multiple regression model taking geomagnetic activity into account. Therefore the third regression model can be expressed as

$$
\begin{aligned}
f_{0} F 2_{h, m}= & c_{0 h, m}+c_{1 h, m} \cdot R+c_{2 h, m} . \\
& \cdot R^{2}+c_{3 h, m} \cdot A p \cdot R+c_{4 h, m} . \\
& \cdot A p+c_{5 h, m} \cdot A p^{2}
\end{aligned}
$$

where Ap is twelve-month running mean values. $c_{0}$ to $c_{5}$ are coefficients at given local time $\mathrm{h}$ for different month $\mathrm{m}$, in which $\mathrm{c}_{3}$ represents of coaction of solar and geomagnetic activity, while $c_{4}$ and $c_{5}$ are the geomagnetic activity amplitudes.

Figure 3 illustrates the diurnal variations of standard deviations for these three regression fitted models from observed values, which shows that, in general, a second-degree regression of $\mathrm{R}$ gives a much lower standard deviations than the linear fit. The significant decreases in standard deviation occur in May from 0.9 to 0.4 and in October from 1.2 to 0.7 . Over Chongqing, the solar cycle dependence of foF2 is obviously nonlinear, especially at 


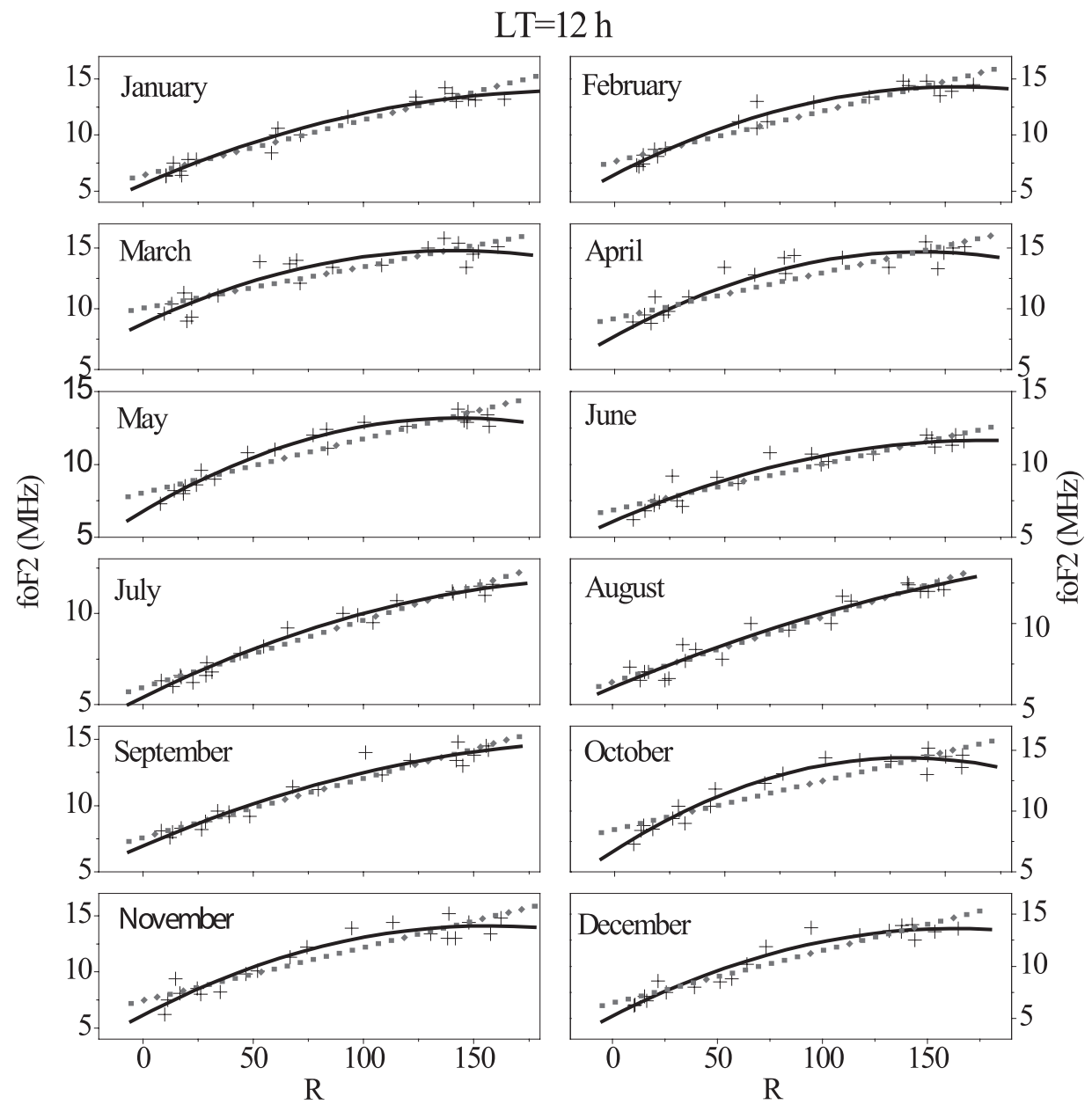

Fig. 1. The responses of the monthly median foF2 to solar activities represented by smoothed monthly mean sunspot number R at local time $12 \mathrm{~h}$ during the whole interval of 1977-1997 over Chongqing. Observed data are plotted with cross. Dotted and solid lines represent the linear and quadratic fits, respectively.

daytime in each month and at nighttime in summer, which supports the conclusion of Kouris and Nissopoulos (1994). Moreover, Liu et al. (2004) obtained similar conclusions using the solar activity index F10.7. On the other hand, when we take geomagnetic activity into account, the standard deviations are smaller than only considering the nonlinear influence of solar activity, but its effect depends on time and month. Generally, a decrease about $0.1 \mathrm{MHz}$ is obtained at daytime. At nighttime, the two model standard deviation is nearlythe same except in summer, in which the standard deviations decrease about 0.2 $\mathrm{MHz}$ using equation (3.3). Over Chongqing, the influence of magnetic activity on foF2 is evident at daytime in each season, and at nighttime in summer. 


\section{Model and results}

Since there are diurnal periodicities inherent in all the geophysical data, Fourier expansion generally has the highest priority in constructing empirical models. The diurnal variations can be well expressed by a Fourier expansion of cosine and sine functions with periods of 24 hours and higher harmonics, and many ionospheric empirical models are established with Fourier expansion (e.g. Zolesi et al., 1993; De Franceschi and Desantis, 1994; Pancheva and Mukhtarov, 1996; Liu et al., 2004). In this way, the diurnal variation of foF2 can be expressed as

$$
\begin{aligned}
f \circ F 2(h, m)= & c_{0, m}+\sum_{i=1}^{N}\left(A_{i, m} \cos \frac{2 \pi}{24} i h+\right. \\
& \left.+B_{i, m} \sin \frac{2 \pi}{24} i h\right)
\end{aligned}
$$

where $\mathrm{h}$ is local time and $\mathrm{N}=6$ is the harmonic number (24-, 12-,8-,6-,4.8- and 4-hour) and $\mathrm{T}$ is equal to 24 hours. These spectral coefficients are a function of $\mathrm{R}$ and Ap. In this model, these coefficients are approximately expressed as equation (3.3), and can be estimated with a least squares regression analysis for the data set in a specified month $\mathrm{m}$.
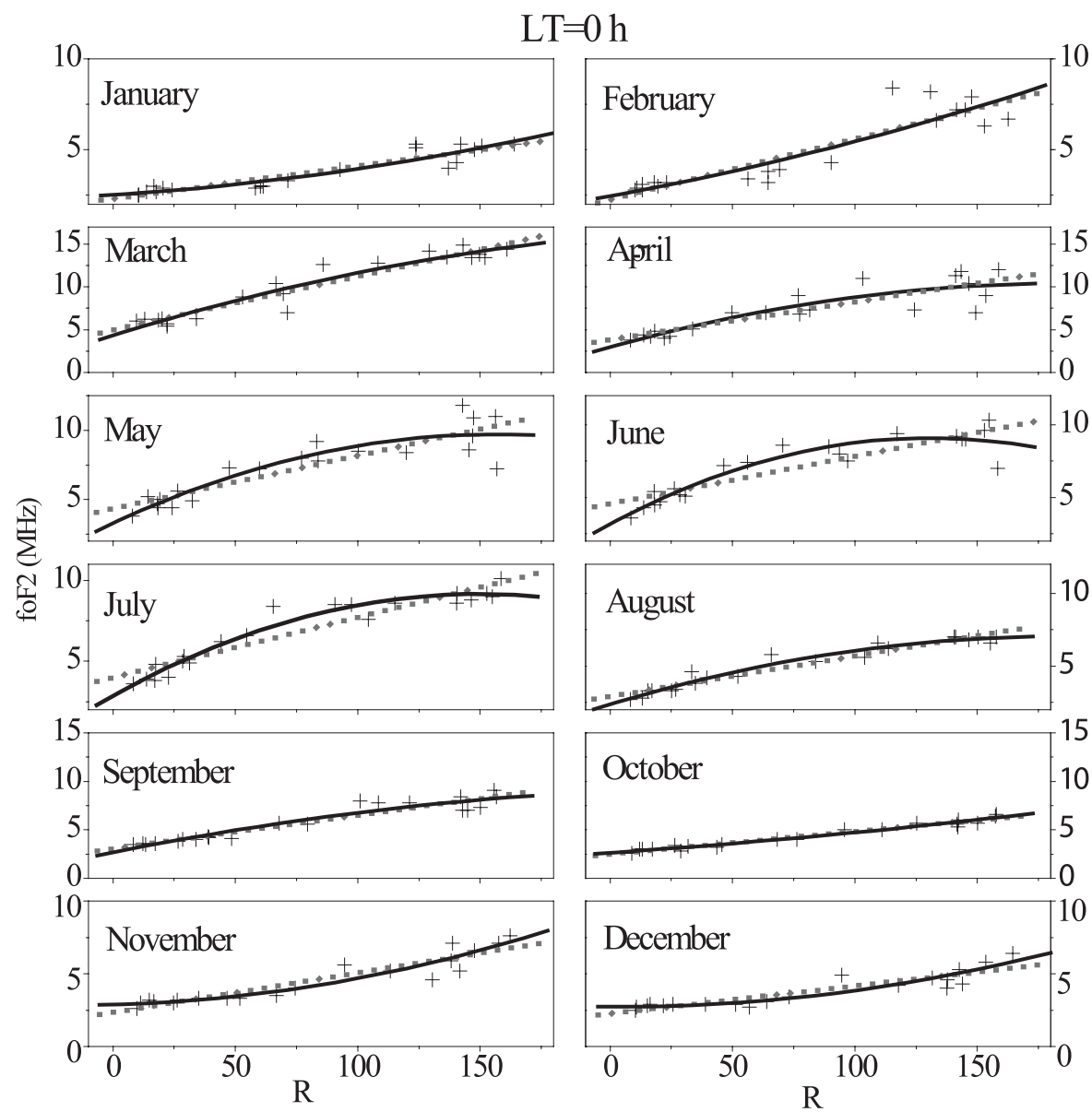

Fig. 2. Same as fig. 1, but for local time $0 \mathrm{~h}$. 


$$
\begin{aligned}
& \longrightarrow \mathrm{foF} 2=\mathrm{a}_{0}+\mathrm{a}_{1} \mathrm{R} \longrightarrow \mathrm{O}-\mathrm{foF} 2=\mathrm{b}_{0}+\mathrm{b}_{1} \mathrm{R}+\mathrm{b}_{2} \mathrm{R}^{2} \\
& \longrightarrow \mathrm{foF} 2=\mathrm{c}_{0}+\mathrm{c}_{1} \mathrm{R}+\mathrm{c}_{2} \mathrm{R}^{2}+\mathrm{c}_{3} \mathrm{RAp}+\mathrm{c}_{4} A p+\mathrm{c}_{5} A p^{2}
\end{aligned}
$$
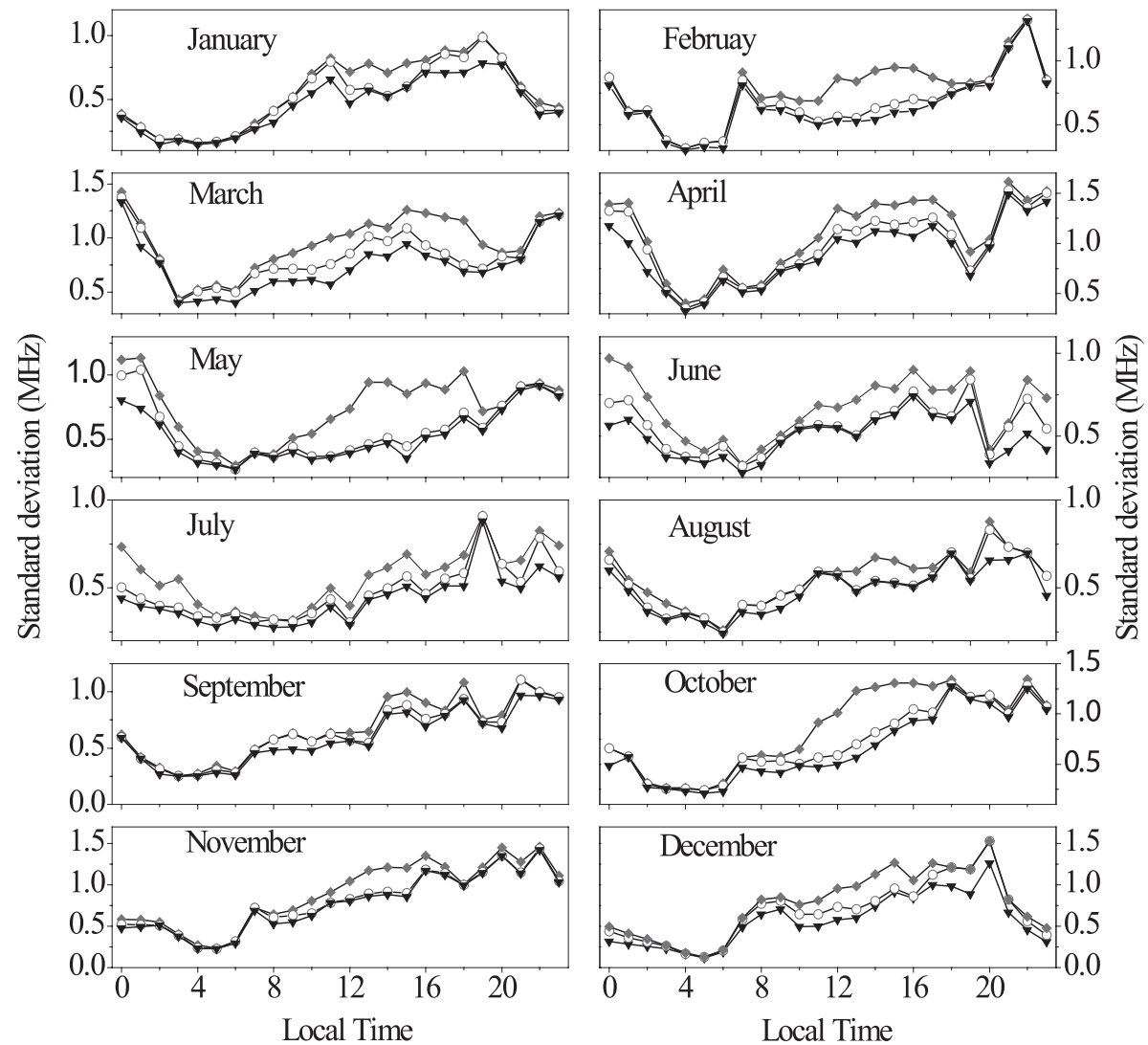

Fig. 3. Diurnal variation of standard deviations of the values of three regression fits form observed foF 2 for the whole interval of 1977-1997 over Chongqing.

The time series of monthly median hourly values of foF2 during the interval 1977-1997 was processed. Figure 4 demonstrates the comparison between our model and the observed data. Six years in which solar activity was high (1980 and 1991), in the ascending part of the solar cycles 21 and 22 (1978 and 1988) and in the descending part of the two cycles (1983 and 1994) are chosen. Furthermore the years of high activity (2000) and descending part of the solar cycle 23 (2003) are also plotted in fig. 4 to illustrate the model ability in long-term prediction. It is obvious that the model describes the diurnal variation of foF2 successfully.

The SSM is compared with IRI by statistic errors shown in fig. 5 and fig. 6 . Figure 5 shows the yearly and diurnal variations of the standard deviations of the model and IRI from the observed values for the whole interval 1977-1997. Evidently this model is better than IRI, and the standard deviations almost decrease about $0.3 \mathrm{MHz}$ for both yearly and diurnal variations with our model compared with IRI except the yearly variations in 1981 and 1988. Figure 6 shows the probability density histogram of foF2 errors with relative deviations from the obser- 
$\odot$ observed data
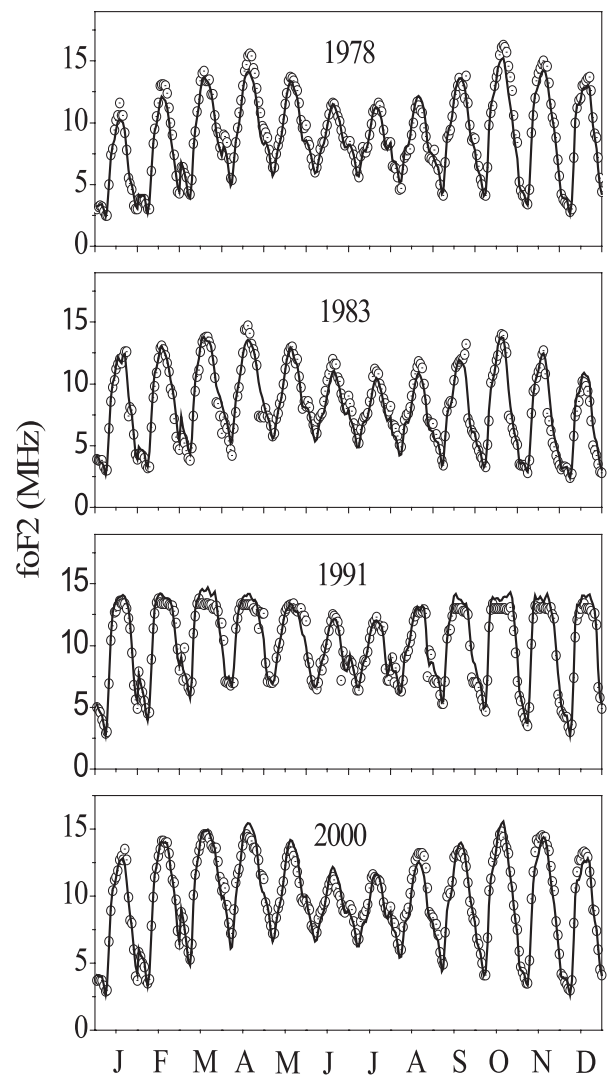

— model values
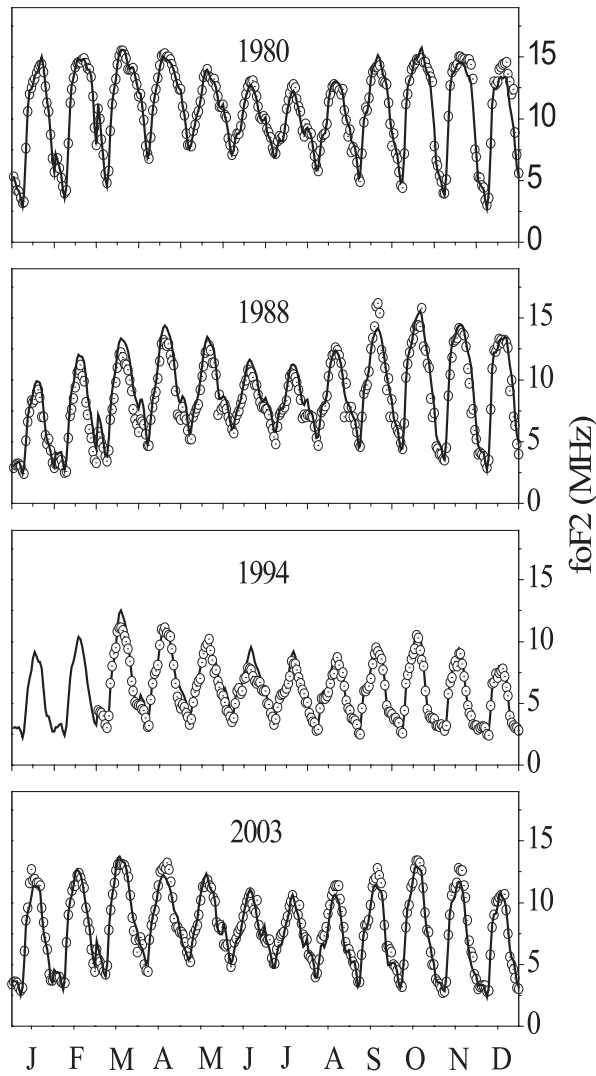

Fig. 4. Comparison between observed (open circles) and modeled (lines) foF 2 values for high solar activity $(1980,1991)$, the descending part of the solar cycle $(1983,1994)$, the ascending part of solar cycle $(1978,1988)$, high solar activity (2003) and descending part of solar cycle 23 (2003) for long-term prediction.

vations. The error distribution is evidently normal which is reasonable. The solid thin line is the Gaussian curve through least squares fit. As Pancheva and Mukhtarov (1996) processed, the relative deviations are not lager than $12 \%$ at the confidence level of $95 \%$ for the SSM, while the errors are about $22 \%$ at the same confidence for IRI model. Moreover, the standard deviation of the model from observations for the whole data set is 0.65 while is 0.96 for IRI model. Thus our new SSM provides a higher accuracy than IRI model. Figures 4-6 have basically demonstrated the advantage of our model.

\section{Discussions and conclusions}

Because global models may smear out features unique to a particular region, single-station models are very useful and widely viewed as the milestone of any ionopheric services (Holt et al., 2002, Pancheva and Mukhtarov, 1998; Liu et al, 2004), and there is no stationspecific model over Chongqing, we developed a SSM of monthly median foF2 based on the observations from 1977 to 1997 . In our model, we chose twelve-month running mean values of sunspot number $\mathrm{R}$ and geomagnetic index Ap 

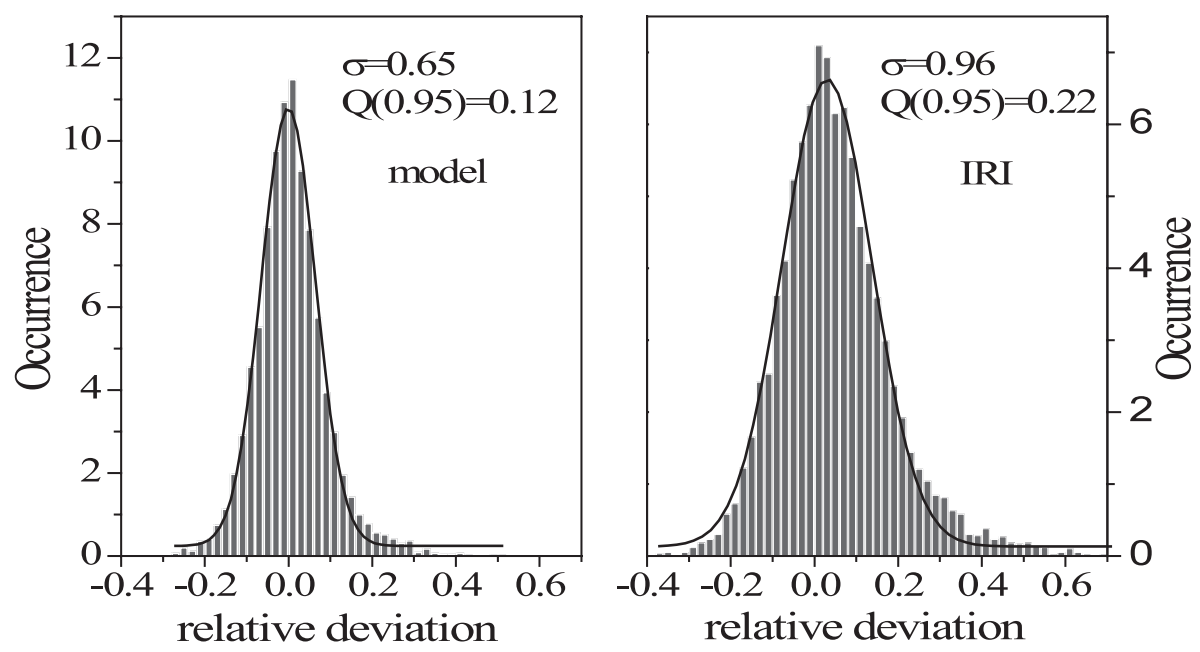

Fig. 5. The standard deviations $(\sigma)$ and the distributions of relative deviation of the new SSM values and IRI model from observed foF2 over Chongqing during the whole interval of 1977-1997. The thin lines are Gaussian fitting curves.

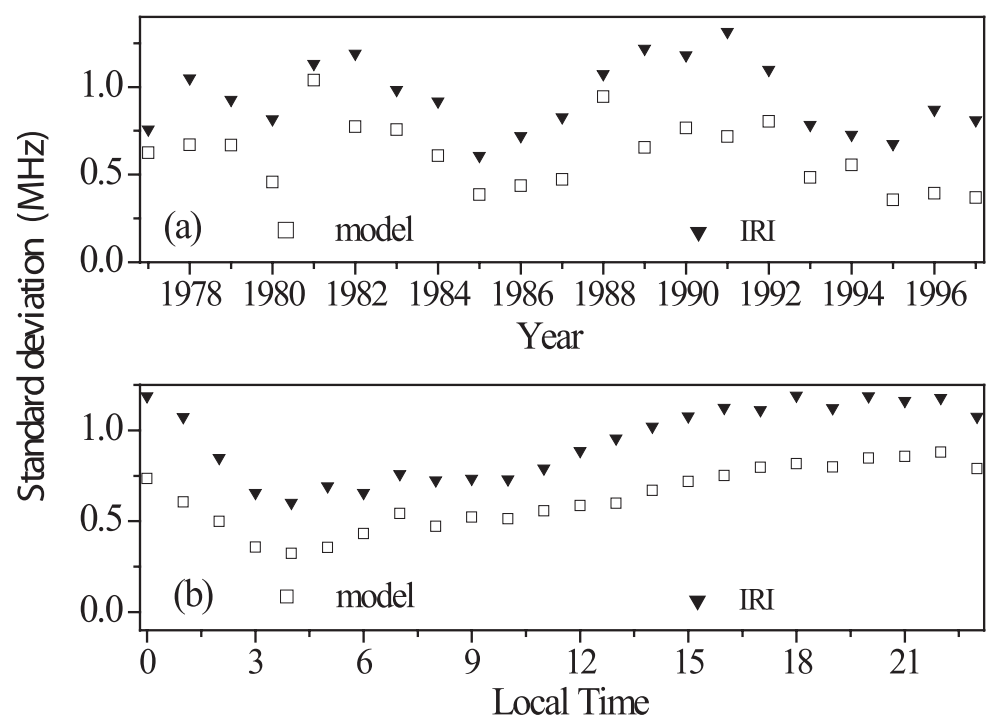

Fig. 6a,b. The (a) yearly and (b) diurnal variations of standard deviations of the SSM and IRI model values form observed foF2 for the whole interval of 1977-1997 over Chongqing.

rather than monthly average or monthly median indices. In practice, the latter may present the influence of solar or geomagnetic activity on ionopheric average action more effectively in specific month, and some empirical models chosen these indices (e.g. Zolesi et al., 1993; 
Liu et al, 2004), nevertheless the indices are difficult to predict with a long lead time because of their severe variations. But the former are relatively smoother and have the advantages of availability, reliability and predictability.

The results of this paper show that there is a nonlinear relationship between monthly median foF2 and solar activity. A second-degree fit gives a much better correlation than the linear fit dependent on hour and month. The standard deviations decrease significantly mostly during daytime in each season and nighttime in summer. Moreover, introducing geomagnetic index Ap further improves the description of variation of monthly median foF 2 . We conclude that the variation of foF 2 is nonlinearly dependent both on solar activity and geomagnetic activity, at Chongqing station, China. Considering both nonlinear influence of solar activity and geomagnetic activity, our model are successfully constructed using Fourier expansion, which is verified with standard deviation and relative deviations distributions compared with IRI model. The SSM has a low standard deviation is $0.65 \mathrm{MHz}$ and its relative deviations are not lager than $12 \%$ at the confidence level of $95 \%$, while standard deviation is $0.96 \mathrm{MHz}$ and the errors are about $22 \%$ at the same confidence for IRI model. Furthermore, the approach of our model could be used for the short-term prediction and disturbance.

\section{Acknowledgements}

The authors are grateful to the anonymous referees for helpful comments. This work is supported by NSFC (National Nature Science Foundation of China) under Grant No.40310223, the National Key Laboratory of Electromagnetic Environment (LEME) under Grant No.9140C0801060803 and by the National Key Technology R\&D Program under Grant No.2006BAB18B06.

\section{REFERENCES}

Apostolov, E., L. Alberca and D. Pancheva (1994): Longterm prediction of the foF 2 on the rising and falling parts of the solar cycle, Adv. Space. Res. 14, 47-50.
Balan, N., G.J. Bailey, B. Jenkins, P.B. Rao and R.J. MOFFETT (1994): Variations of ionospheric ionization and related solar fluxes during an intense solar cycle, $J$. Geophys. Res., 99, 2243-2253.

BilitZA, D. (2000): The importance of EUV indices for the International Reference Ionosphphere, Phys. Chem. Earth., 25, 515-521.

BiLiTZA, D. (2001): International reference ionosphere 2000, Radio Sci., 36, 261-275.

BiLitZA, D. (2002): Ionospheric models for radiao propagation studies, in the review of Radio Science 1999-2002 edited by W. R. STONE, IEEE Press, 625-679.

Caruana, J. (1990): The IPS monthly T-index, Proc. of the solar-Terrestrial Prediction Workshop, Leura, Australia, 2, 257-263.

De Franceschi G. and A. Desantis (1994): PASHA: regional long term predictions ionospheric parameters by ACHA, Ann. Geophysics, 37, 209-220.

DOMINICI, P. and B. ZOLESI (1987): A model for the normal ionosphere over Rome, II Nuovo Cimento, 10C, 191208.

Dick, M. and P. BRADLEY (1992): The RAL multi-quasiparabolic model ionosphere, Porc. of PRIME Workshop, Roquetes, Memoria, 16, 67-83.

Forbes, J. M., S. E. PALO and X.L. ZHANG (2000): Variability of the ionosphere, J. Atmos. Terr. Phys., 62, 685693.

Holt, J. M., S. R. Zhang and M. J. Buonsanto (2002): Regional and local ionospheric models based on Millstone Hill incoherent scatter radar data, Geophys. Res. Lett. 29, 1027.

HuANG, Y.N. (1963): The hysteresis variation of the semithickness of the F2-layer and its relevant phenomena at Kokubunji, Japan, J. Atmos. Terr. Phys., 25, 647-658.

KANE, R.P. (1992): Solar cycle variation of foF2, J. Atmos. Terr. Phys., 54, 1201-1205.

Kouris, S.S., Th.D. Xenos, and S. Goutzamanis (1993): Proposals for the solar-cycle variations of foF2, Porc. of PRIME Workshop, Graz. Wissenschaftlucher Bericht, 2, 79-88.

Kouris, S.S. and J.K. Nissopoulos (1994): Variation of foF2 with solar activity, Adv. Space Res., 14, 163-166.

Kouris, S.S., P.A. Bradley and P. Dominici (1998): Solarcycle variation of the daily foF2 and M(3000)F2, Ann. Geophysicae, 16, 1093-1042.

LiU, J. Y., Y. I. CHEN and J. S. LIN (2003): Statistical investigation of the saturation effect in the ionospheric foF2 versus sunspot, solar radio noise, and solar EUV radiation, J. Geophys. Res., 108, 1067-1073.

LIU, L.B., W.X. WAN and B.Q. NING (2004): Statistical modeling of ionospheric foF2 over Wuhan, Radio Sci., 39, 2013.

LIU, R., P. Smith and J. KING (1983): A new solar index to improve foF 2 prediction using the CCIR, Altas. Telecomm. J. 50, 408-413.

Mikhailov, A. and V. Mikhailov (1995): A new ionospheric index MF2, Adv. Space. Res., 15, 93-97.

Moraitis, G., Z. KeCIC and L. CANDER (1991): Ionospheric modeling at single stations, Porc. of PRIME Workshop, Rome, 154-160.

PancheVA, D. and P. Mukhtarov (1996): A single-station spectral model of the monthly median F-region critical frequency, Ann. Geophysics, 39, 807-818. 
Pancheva, D. and P. Mukhtarov (1998): A single-station spectral model of the monthly median foF2 and M(3000) F2, Studia geoph. et geod., 42, 183-196.

RAO, M.S. and R.S. RAO (1969): The hysteresis variation in F2 layer parameters, J. Atmos. Terr. Phys., 31, 11191125.

RICHARDS, P.G. (2001): Seasonal and solar cycle variations of the ionospheric peak electron density: Comparison of measurement and models, J. Geophys. Res., 106, 12803-12819.

Sethi, N.K., M.K. Goel and K. K. Mahajan (2002): Solar cycle variations of foF2 from IGY to 1990, Ann. Geophysicae, 20, 1677-1685.

SizUN, H. (1991): foF2 variation with R12 solar index, Proc. of PRIME Workshop, Rome, 87-91.

Sizun, H. (1992): F2-layer critical frequency modelization as a function of upward and downward phase of solar activity cycle, Proc. of PRIME/URSI Workshop, Roquetes, Memoria, 16, 205-217.
SoLÉ, J.G. (1988): Relations between hourly monthly median values of foF2 and some geophysical indices. Their application to an ionospheric single station model, Acta Geophys. Polonica, 17, 77-88.

Xenos, Th.D., S.S. Kouris and B. Zolesi (1996): Assessment of the solar-cycle dependence of foF2, Ann. Geophysics, 6, 1157-1166.

Zhang, M.L., J.K. SHI, X. WANG and M. Radicella (2004): Ionospheric variability at low latitude station: Hainan, China, Adv. Space. Res., 34, 1860-1868.

Zolesi, B., Lj.R. CANDER and G.D. Franceschi (1993): Simplified ionospheic regional model for telecommunication application, Radio Sci., 28, 603-612.

Zolesi, B. and Lj.R. CANDER (2000): Evolution of the ionospheric mapping and modeling during the last four decades, Fisica de la Tierra, 12, 127-154.

(received November 21, 2007; Accepted May 9, 2008) 